\title{
Expectant Management of Placenta Accreta Spectrum Disorders
}

\author{
Eiji Kondoh, MD, PhD ${ }^{1}$ \\ ${ }^{1}$ Department of Gynecology and Obstetrics, Kyoto University, \\ Kyoto, Japan \\ Surg J (NY) 2021;7(suppl S1):S2-S6.
}

\begin{abstract}
Address for correspondence Eiji Kondoh, MD, PhD, Department of Gynecology and Obstetrics, Graduate School of Medicine, Kyoto University, 54 Shogoin Kawahara-cho, Sakyo-ku, Kyoto 606-8507, Japan (e-mail: kondo@kuhp.kyoto-u.ac.jp).
\end{abstract}

\author{
Abstract \\ Keywords \\ - balloon tamponade \\ - expectant \\ management \\ - leaving the placenta \\ in situ \\ - placenta accreta \\ spectrum \\ - uncontrolled \\ hemorrhage
}

Placenta accreta spectrum (PAS) disorder is a potentially life-threatening complication. The incidence of PAS has increased over the past few decades, mainly due to the increased cesarean section rate. While cesarean hysterectomy remains the most standard treatment for the management of PAS, expectant management is becoming increasingly prevalent to avoid serious maternal morbidity and maintain future fertility. Expectant management is defined as leaving the placenta either partially or fully in situ, and waiting for its spontaneous resorption or expulsion. The success rate of expectant management is high, but intraoperative uncontrolled hemorrhage results in hysterectomy. Moreover, some individuals fail expectant management and require delayed hysterectomy due to complications such as secondary postpartum hemorrhage, sepsis, uterine necrosis, and vesicouterine fistula. As a result of the very limited data currently available, there is no consensus on the optimal strategy for the expectant management of PAS. However, it is clear that a multidisciplinary team approach in tertiary centers is essential for women with PAS. In addition, meticulous preparation is the key to successful expectant management. Here, we describe a surgical strategy designed to reduce perioperative blood loss, which is a minimum requirement to ensure maternal safety. This article also addresses practical issues in expectant management of PAS, based on the published literature and our own experience.
The primary cause of placenta accreta spectrum (PAS) is a lack of decidua basalis defect, and risk factors for PAS include placenta previa, uterine curettage, myomectomy, and advanced maternal age. ${ }^{1}$ Ultrasound evaluation, with gray-scale and complementary color Doppler imaging, is useful for the diagnosis of PAS. The presence of placental lacunae with turbulent flow (-Fig. 1) is highly suggestive of PAS, though normal ultrasound findings do not exclude a diagnosis of PAS. ${ }^{2}$ Magnetic resonance imaging (MRI) may improve the diagnosis of PAS located in the posterior wall where ultrasound findings are inconclusive., ${ }^{2,3}$ In addition, MRI can help to plan surgical strategies for PAS, as it can provide more comprehensive and detailed information about the location of the placenta, the uterus, and the adjacent organs. ${ }^{3}$

The antenatal diagnosis of PAS provides an opportunity for in-depth planning and a multidisciplinary team approach. The average perioperative blood loss for PAS is $\sim 3,000$ to $5,000 \mathrm{~mL}^{3}$ PAS should be managed in a facility that is capable of massive blood transfusion. Preoperative autologous blood donation and intraoperative cell salvage should be considered. It is recommended to plan a cesarean section whenever possible. The optimal timing of delivery remains controversial and should be individualized according to the presence of warning signs such as vaginal published online

June 3, 2021
DOI https://doi.org/ 10.1055/s-0040-1722240. ISSN 2378-5128.

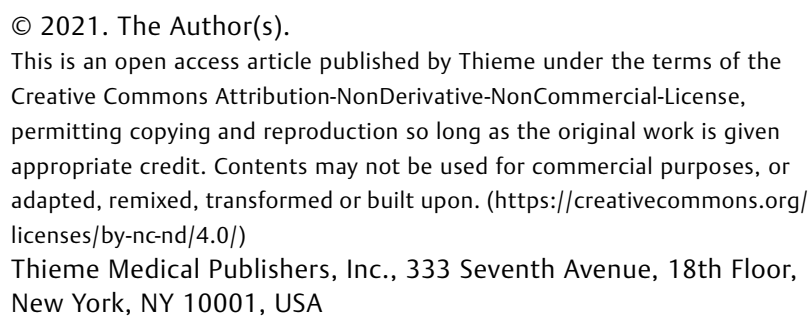




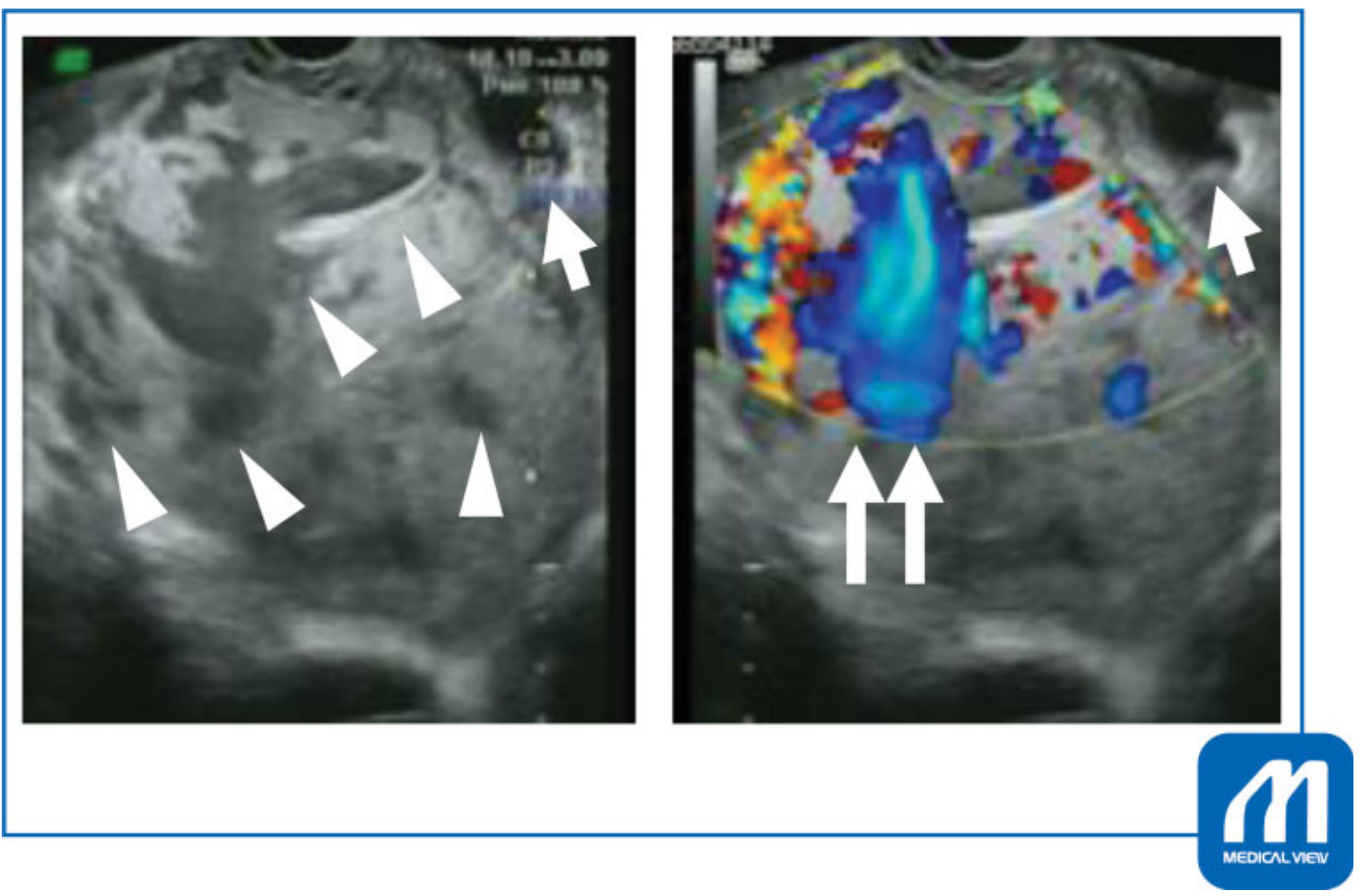

Fig. 1 Transvaginal ultrasonography showing placenta percreta with urinary bladder involvement. The gray-scale ultrasound imaging (left) shows multiple irregular placental lacunae (arrowheads), and the color Doppler image (right) demonstrates with turbulent flow in the lacunae (double arrows). The arrow indicates the bladder. (Reproduced with permission from Eiji Kondoh. In: Hiramatsu Y, Konishi I, Sakuragi N, Takeda S, eds. OGS NOW, No.22. (Japanese). Tokyo: Medical View; 2015:59. Copyright @ Medical View).

bleeding or sonographic cervical length. Increased risk of maternal profuse hemorrhage should be balanced with the risk of neonatal prematurity. If an antenatal diagnosis of PAS is obvious, the scheduled delivery at $340 / 7$ to $356 / 7$ weeks of gestation is reasonable and acceptable. ${ }^{2}$ If possible, a cesarean section should be performed in a hybrid operating room so that interventional radiology can be performed as needed. ${ }^{2,3}$

\section{Surgical Steps}

\section{Anesthesia}

Large indwelling catheters should be used to secure multiple venous lines, and an arterial catheter should be placed to monitor hemodynamics. The choice of an anesthetic technique (spinal or general anesthesia) must be made by an anesthesiologist. General anesthesia is usually preferred in cases with a high risk of massive hemorrhage and coagulopathy. However, false positive rates for the antenatal diagnosis of PAS range from 18 to $28 \%^{3}$ Moreover, if an approach involving leaving the placenta in situ is successfully performed without spontaneous placental separation, blood loss is minimized and the patient remains hemodynamically stable. Therefore, the choice of anesthesia should be individualized, depending on each patient's circumstances (e.g., the level of placental invasion and its location).

\section{Surgical Position}

Supine split-leg position is preferred because intraoperative vaginal bleeding can be directly viewed.

\section{Urethral Stent Placement}

A prophylactic ureteral stent helps to reduce iatrogenic ureteral injury when hysterectomy is needed.

\section{Occlusion Balloon Catheter Placement (Common Iliac Artery or Infrarenal Abdominal Aorta)}

Intraoperative hemorrhage should be well controlled. Therefore, the use of prophylactic artery occlusion catheters is strongly encouraged if bladder involvement is highly suspected. ${ }^{3}$ It is better to measure radiation dose to minimize exposure to the fetus. As fetal bradycardia can occur due to subsequent spasm of the uterine artery, fetal heart rate monitoring is recommended during a catheterization. Pulse oximeters are worn on both innermost toes to assess the degree of blood flow blockade ( - Fig. 2). There are conflicting reports regarding optimal balloon locations. ${ }^{3}$ Some studies reported that balloon occlusion in the internal iliac arteries reduced intraoperative blood loss, but most studies noted that balloon occlusion in the internal iliac arteries has only limited effects on reducing blood loss, probably due to the extensive network of collateral vessels. ${ }^{3}$ Therefore, it should be chosen to block blood flow at the location of the common iliac artery or renal inferior abdominal aorta. 


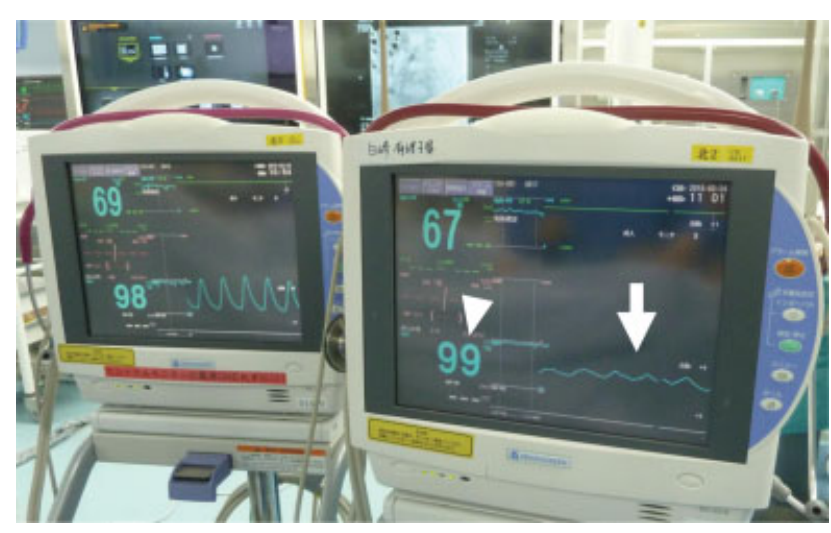

Fig. 2 Pulse oximeter waveform. Pulse oximetry waveforms after a temporary blockage of the flow of one common iliac artery. The display on the left shows a normal pulse oximetry waveform and right shows a low amplitude waveform (arrow), indicating poor leg perfusion. Note that $\mathrm{SpO}_{2}$ measurements are kept at 99\% (arrowhead). (Reproduced with permission from Eiji Kondoh, Ikuo Konishi. Perinatal Medicine (Tokyo). (Japanese). Tokyo Igakusha. 2015;45:1131-1135).

\section{Laparotomy and Close Observation}

A midline incision is preferred as a laparotomy approach for PAS. The uterine surface and the uterine serosa-bladder interface should be carefully inspected for PAS findings. The representative intraoperative appearance of PAS includes placenta bulging through the uterine wall and hypervascularity of the interface between the uterine wall and the bladder.

Intraoperative sonography is recommended to determine the site of uterine incision, which should be made away from the placental edge. The maximum degree of placental invasion and its area should also be re-evaluated before the following procedure.

\section{Bladder Separation}

If bladder involvement is unclear, the bladder can be carefully separated from the uterus. This allows for a U-shaped suture to control heavy bleeding when the placenta is partially detached.

\section{Hysterotomy and Cesarean Delivery}

If the placenta covers the anterior wall extensively, a transfundal uterine incision is selected. In other cases, the incision site is selected flexibly by using a vertical incision or a transverse incision higher than usual to avoid cutting through the placenta.

\section{Minimizing Perioperative Blood Loss}

Attempts at forced placental removal are strictly prohibited because this causes massive hemorrhage. ${ }^{2,3}$ However, if an antenatal diagnosis of PAS is uncertain and there is no definite operative finding suggestive of PAS, the patient may not have actually PAS. In addition, the placenta is often partly separated spontaneously, causing excessive bleeding. Therefore, uterotonic agents should be administered regularly in all cases to check for spontaneous partial or complete uterine placenta separation and to reduce blood loss. ${ }^{3}$ Moreover, gentle umbilical traction is an accepted approach, especially in the case of ambiguous PAS findings. The attempt can avoid unnecessary complications such as delayed hemorrhage, infection, and coagulopathy, and longterm surveillance in expectantly managed false-positive PAS. $^{3}$

How to deal with hemorrhage depends on the degree of spontaneous detachment of the placenta. ${ }^{3}$ If the placenta is completely separated from the uterus, the actual diagnosis is placenta previa, which can be safely managed with intrauterine balloon tamponade. If the placenta is completely attached to the uterus, an approach involving leaving the placenta in situ will minimize blood loss in cesarean section. If the placenta is partially separated from the uterus, severe hemorrhage will occur. First, the gauze is firmly pressed onto the bleeding site with both hands. Then, the balloon of the artery is inflated and it is confirmed that the pulse oximeter waveforms of both toes are attenuated. The spontaneously separated part of the placenta is ligated and cut off. Several U-shaped sutures placed through the placenta and the anterior wall of the uterus together help to clinically reduce blood volume. Intrauterine balloon tamponade is particularly useful as an adjunct intervention. ${ }^{4}$ When the placenta completely covers the uterine ostium, the balloon shaft is taken out outside the body through the uterus and abdominal wall (-Fig. 3). ${ }^{4}$ Even before expansion, the Bakri balloon protrudes from the diameter of the shaft and the balloon cannot be removed from the abdomen afterwards. Therefore, multiple 22 Fr Foley catheters are used as a modified method. ${ }^{4}$ If bleeding cannot be controlled by the hemostatic methods above, arterial embolization is performed. Hysterectomy is also a reasonable option.

\section{Uterine Closure}

The blood flow block is released and hemostasis is confirmed. When the modified intrauterine balloon tamponade is performed, the drain is placed intraperitoneally. The interventional radiology sheath is left for 24 hours after surgery to prepare for massive bleeding. The intrauterine balloon will also be removed the day after surgery.

\section{Postoperative Management}

Careful follow-up is required due to the potential for massive bleeding, infections, sepsis, and blood clotting disorders in the long-term. ${ }^{2-5}$ Patients and their families need to have the currently known risks explained, and must understand the follow-up methods and their importance, as management methods are not yet established. Regarding discharge, patients can be discharged according to the normal criteria of the center. In the largest series, 78\% (131/167) did not require hysterectomy, and spontaneous placental resorption was observed in $75 \%$ of cases (87/116) with a careful waiting and observation approach. ${ }^{6}$ Moreover, planned preventive delayed interventions, such as hysterectomy, hysteroscopic resection, dilation and curettage, and manual removal of the placenta, can lead to large amounts of unexpected bleeding 

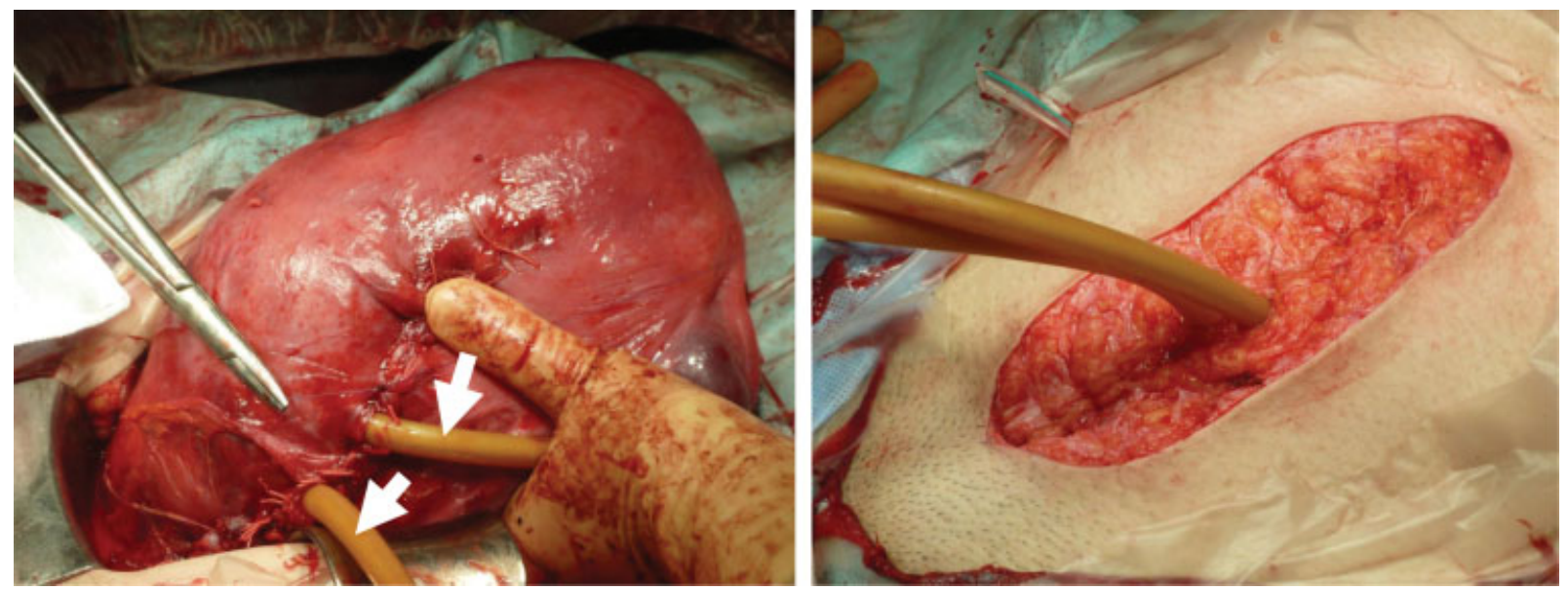

Fig. 3 Modified intrauterine balloon tamponade. The shafts (arrow) of Foley balloon catheters were taken out through a uterine incision (left) and the abdominal wall (right). - Fig. 3 (left): Reproduced with permission from Eiji Kondoh, Ikuo Konishi. Perinatal Medicine (Tokyo). (Japanese). Tokyo Igakusha 2015;45:1131-1135. - Fig. 3 (right): Reproduced with permission from Eiji Kondoh, Kaoru Kawasaki, et al. Successful management of intraoperative hemorrhage from placenta previa accreta: intrauterine tamponade balloons brought out through the abdominal wall. J Matern Fetal Neonatal Med Taylor \& Francis. 2014;27(3):309-311.

or damage to adjacent organs. $2,3,5$ Thus, intervention for retained placenta is not recommended for uncomplicated patients. ${ }^{2,3,5}$

We think that massive bleeding can occur as long as the placental blood flow remains. In a small case series, placental blood flow disappeared on average in $8.9 \pm 1.7$ weeks, and the loss of placental blood flow was almost coincident with the reduction in human chorionic gonadotropin (hCG) to undetectable levels. ${ }^{7}$ Therefore, color Doppler ultrasonography and serum hCG measurements may help to monitor patients to assess the potential risk of delayed hemorrhage. Attempts at prophylactic embolization to prevent bleeding are controversial. ${ }^{2,3,5}$ Uterine artery embolization carries the risk of serious complications such as uterine necrosis and refractory infections. ${ }^{3,6}$ Interestingly, there was no significant difference in the timing of hCG dropping to undetectable levels, placental blood flow loss, and placental disappearance, regardless of the use of embolization and the amount of early residual placenta. ${ }^{7}$ Therefore, we have not advocated that prophylactic embolization be performed.

It is not uncommon to observe sudden high fever or elevated inflammatory responses during follow-up. ${ }^{3,5,6}$ Although infections are usually cured with antibiotics, infected necrotic uterus after embolization is resistant to antibiotic therapy and often requires hysterectomy. During follow-ups, internal examinations should be avoided as much as possible to prevent ascending infections. Coagulopathy is rare, but can occur with expectant management. ${ }^{3,5,6}$ Therefore, regular follow-up tests include ultrasonography, a speculum examination, complete blood count, C-reactive protein, hCG, and coagulation tests (prothrombin time, activated partial thromboplastin time, fibrinogen). Follow-up is set weekly and then biweekly to monthly. After the loss of placental blood flow or an undetectable level of hCG, intervals are every few months to confirm that the placenta has disappeared. Follow-up ends when the menstrual cycle resumes.

Methotrexate has long been used as an adjunct therapy for expectant management. However, methotrexate acts only on actively dividing cells, whereas trophoblast cells rarely proliferate during late pregnancy and after childbirth. Thus, the use of methotrexate to accelerate placental absorption is not recommended. $2,3,5$

\section{Tips and Warnings}

The biggest obstacle to attempting expectant management is massive hemorrhage that occurs when the placenta is partially detached. ${ }^{3,4}$ Before surgery, we need to repeatedly visualize in our mind how to overcome intractable bleeding until we are satisfied. U-shaped suture followed by balloon tamponade is effective in controlling bleeding. ${ }^{4}$ However, if the bleeding cannot be controlled, hysterectomy should be undertaken promptly without sticking to expectant management. During the first 3 months of observation after surgery, it is important to wait patiently for the placental blood flow to disappear. ${ }^{3-8}$ No additional intervention is needed if the course is uneventful.

Conflict of Interest

None.

\section{References}

1 Jauniaux E, Chantraine F, Silver RM, Langhoff-Roos JFIGO Placenta Accreta Diagnosis and Management Expert Consensus Panel. FIGO consensus guidelines on placenta accreta spectrum disorders: epidemiology. Int J Gynaecol Obstet 2018;140(03):265-273

2 Cahill AG, Beigi R, Heine RP, Silver RM, Wax JRSociety of Gynecologic Oncology American College of Obstetricians and 
Gynecologists and the Society for Maternal-Fetal Medicine. Placenta accreta spectrum. Am J Obstet Gynecol 2018;219(06): B2-B16

3 Kondoh E, Kawasaki K, Chigusa Y, et al. Optimal strategies for conservative management of placenta accreta: a review of the literature. Hypertens Res Pregnancy 2015;3:19-27

4 Kondoh E, Kawasaki K, Kawamura A, Ueda A, Fujita K, Konishi I. Successful management of intraoperative hemorrhage from placenta previa accreta: intrauterine tamponade balloons brought out through the abdominal wall. J Matern Fetal Neonatal Med 2014;27(03):309-311

5 Sentilhes L, Kayem G, Chandraharan E, Palacios-Jaraquemada J, Jauniaux EFIGO Placenta Accreta Diagnosis and Management Expert Consensus Panel. FIGO consensus guidelines on placenta accreta spectrum disorders: conservative management. Int J Gynaecol Obstet 2018;140(03):291-298

6 Sentilhes L, Ambroselli C, Kayem G, et al. Maternal outcome after conservative treatment of placenta accreta. Obstet Gynecol 2010; 115(03):526-534

7 Imai S, Kondoh E, Kawasaki K, et al. Placental blood flow disappears coincident with a fall in human chorionic gonadotropin to undetectable levels in conservative management of placenta accreta. Eur J Obstet Gynecol Reprod Biol 2014; 180:199-201

8 Ueda Y, Kondoh E, Kakui K, et al. Serial magnetic resonance imaging of placenta percreta with bladder involvement during pregnancy and postpartum: a case report. J Obstet Gynaecol Res 2013;39(01):359-363 